\title{
Tunable time delays in photonic-crystal waveguides
}

\author{
M. L. Povinellia ${ }^{\mathrm{a}}$, S. G. Johnson ${ }^{\mathrm{b}}$, and J. D. Joannopoulos ${ }^{c}$ \\ ${ }^{a}$ Ginzton Laboratory, Stanford University, Stanford, CA 94305 \\ ${ }^{\mathrm{b}}$ Department of Mathematics, Massachusetts Institute of Technology, \\ 77 Massachusetts Avenue, Cambridge MA 02139 \\ ${ }^{\mathrm{c}}$ Department of Physics and Center for Materials Science and Engineering, \\ Massachusetts Institute of Technology, 77 Massachusetts Avenue, \\ Cambridge MA 02139
}

\begin{abstract}
We study tunable time-delay devices in which the time delay is tuned by changing the group velocity of the propagating signal. The device is designed to place the operating frequency near a photonic band edge. This enhances the change in delay for a given tuning range of the device refractive index. Here we provide an extended explanation of mode symmetry, nomenclature, and the complete band structure of a sample, integrated device to aid the understanding of our previously published work.
\end{abstract}

Keywords: Integrated optical devices, tunable time delays, photonic-crystal waveguides, slow light

\section{INTRODUCTION}

Delaying an optical signal is useful for a number of applications, including optical buffering, signal processing, logic, radio-frequency (RF) photonics, and enhanced nonlinearities. ${ }^{1}$ Several recent research efforts have focused on replacing relatively bulky fiber-optic delay-line systems ${ }^{2}$ with compact integrated devices. Approaches have included the use of all-pass filters ${ }^{1,3,4}$ and coupled-resonator optical waveguides. ${ }^{5-12}$ Here, we consider an alternate approach to tunable, integrated time delays: slow-light, band-edge photonic-crystal waveguides. Previous work in ideal, 1-D structures has suggested that near a photonic band edge, a large delay tunability can be achieved for a small change in refractive index. ${ }^{13}$ We investigate this slow-light enhancement phenomenon in realistic, three-dimensional structures amenable to on-chip fabrication using established lithographic techniques. In contrast to other work, ${ }^{14,15}$ we use a single waveguide through which light is transmitted at slow speeds near, but not at, the band edge.

In previously published work, ${ }^{16}$ we have described the mechanism of tuning enhancement near a photonic band edge. In addition, we have introduced relevant figures of merit to describe tunable time-delay devices, including sensitivity, length, and dispersion. We have also introduced a quadratic band model to illustrate the dependence of the figures of merit on distance from the band edge and grating strength; sensitivity was seen to increase with decreasing distance to the band edge and also with increasing grating strength. The validity of the quadratic band model was demonstrated by comparison to direct numerical calculations on a realistic, three-dimensional structure. We have also discussed how two grating segments can be cascaded to compensate for dispersion effects and achieve nearly-flat time delays across the operating bandwidth. Moreover, we have demonstrated efficient adiabatic tapering to achieve high transmission into and out of the grated waveguide from a uniform, strip waveguide.

In this manuscript, we clarify the details of the dispersion diagram of the sample device. In particular, we provide a detailed description of mode symmetry in the device and the nomenclature we use to describe it. We also show a complete band structure for the device, including modes with both even- and odd- vector symmetry, and plot their associated field profiles.

Photonic Crystal Materials and Devices IV, edited by Ali Adibi, Shawn-Yu Lin, Axel Scherer,

Proc. of SPIE Vol. 6128, 61280R, (2006) · 0277-786X/06/\$15 · doi: 10.1117/12.646738

Proc. of SPIE Vol. 6128 61280R-1 
(a)

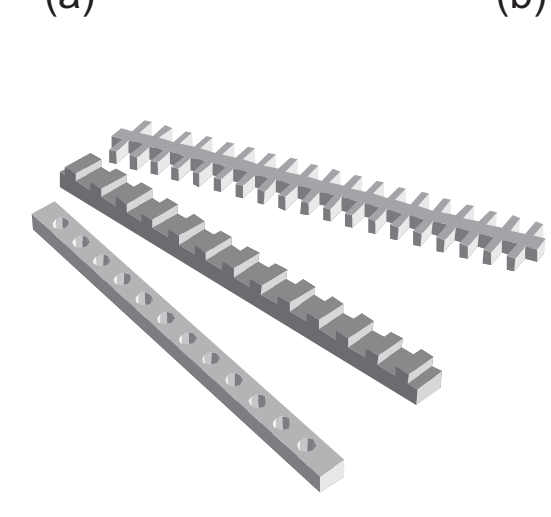

(b)

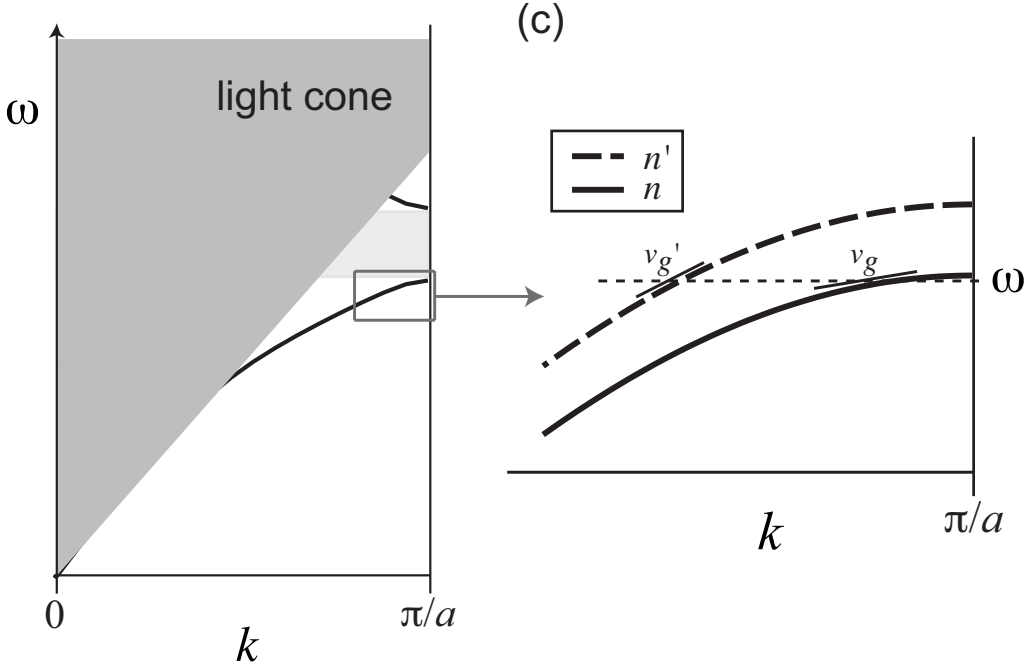

Figure 1. (a) Types of 1-D periodic gratings. (b) Typical band structure of a 1-D periodic grating. The band gap is shaded light gray. (c) Magnified view of the band structure near the band edge, illustrating how a small shift in refractive index can lead to a large shift in group velocity.

\section{PRINCIPLE OF OPERATION}

We are interested in tunable time-delay devices in which a tuning enhancement is achieved by operating near the photonic band edge. The principle of operation has been described in our earlier work, ${ }^{16}$ and is briefly revisited here. Figure 1(a) shows three different types of one-dimensionally periodic gratings, which could for example be fabricated in silicon-on-insulator systems, and would typically rest on a lower-index, oxide substrate. A generic dispersion diagram for gratings of this type is shown in Figure 1 (b), where frequency is plotted as a function of Bloch wave vector. The Bloch wave vector is restricted to values between 0 and $\pi / a$, where $a$ is the periodicity of the grating. The dark grey, shaded region indicates the light cone. Modes with frequency and wave vector in this range are not guided in the grating, but are extended in the surrounding air (above) or oxide (below). Guided modes lie underneath the light cone and are indicated by solid black lines. The lowest-frequency guided mode will typically fold at a wave vector of $\pi / a$, opening up a photonic band gap (shaded, light grey region). A close-up view of the edge of the lowest band near the photonic band gap is shown in Figure 1 (c), which shows the effect of a small index shift. The solid line indicates the guided mode for some initial value of the refractive index of the grating, $n$. The dotted line indicates the guided mode for a slightly shifted value $n^{\prime}$. For small index shifts, the effect on the band is a slight frequency shift. As a consequence, a signal propagating through the device at constant frequency $\omega$ (indicated by the horizontal, dashed line) will experience a shift in group velocity. The group velocity is given by the slope of the dispersion relation $\left(v_{g}=d \omega / d k\right)$, as indicated in the figure. Clearly, for frequencies near the band edge, a small index shift can result in a very large change in group velocity. The change in group velocity correspondingly results in a change in propagation time through the device, yielding a highly-tunable time delay.

\section{PROTOTYPICAL DEVICE}

To make our discussion of a tunable-delay scheme concrete, we have chosen to analyze the particular, realistic device shown in Figure 2 (a) and (b). The device consists of a silicon strip waveguide with grated sidewalls ${ }^{17}$ resting on a silicon-dioxide substrate. Silicon is a particularly favorable material for thermal tuning, since its refractive index varies by more than $1 \%$ from 100 to 300 degrees Celsius. Thermal tuning of the band edge of an integrated silicon device (and consequently of the group index) has recently been demonstrated experimentally in a photonic-crystal slab system. ${ }^{18}$ Moreover, the large refractive index $f$ silicon allows the design of structures with relatively large photonic band gaps. The structural parameters of the device were chosen to maximize the 
(a)

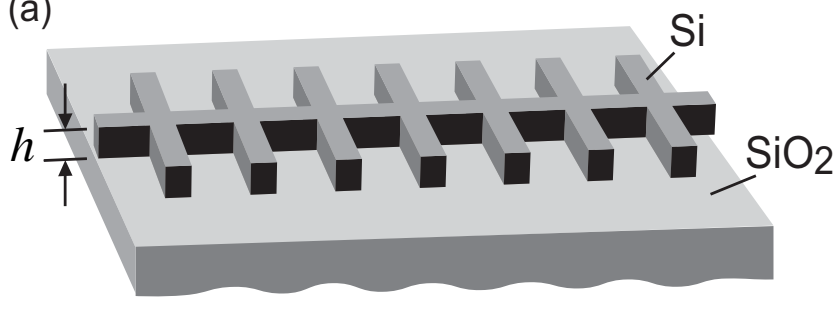

(b)

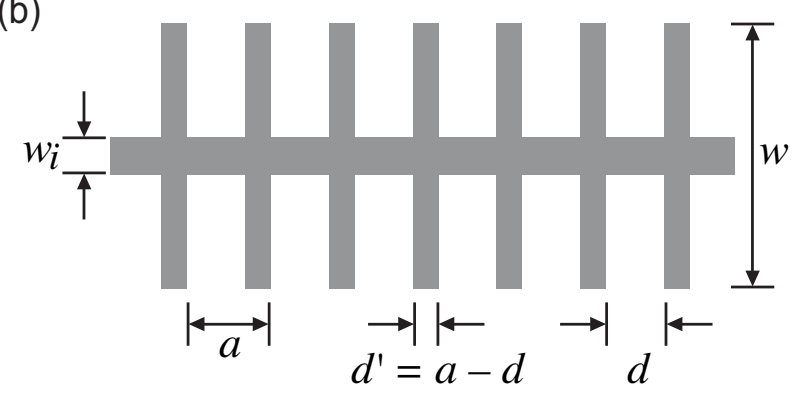

(c)

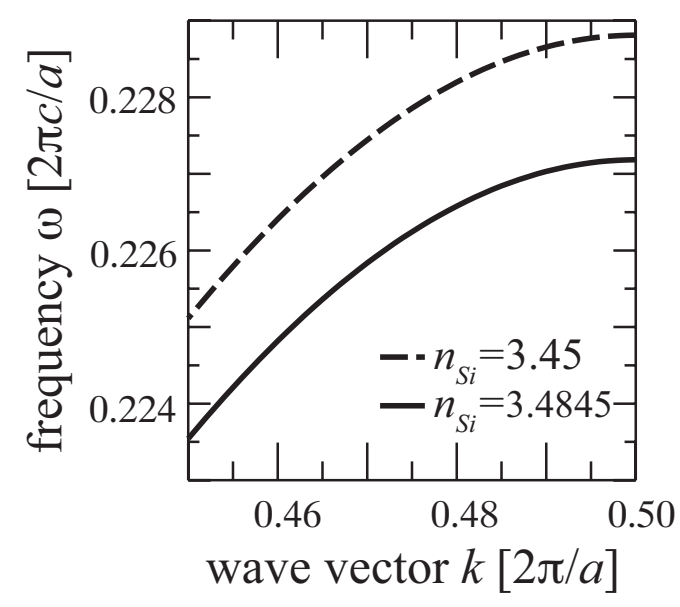

Figure 2. 3-D perspective view of a slow-light grating structure. (b) Top view. (c) Band structure.

size of the first band gap under the constraint that the minimum feature size be at least $0.15 \mu \mathrm{m}$. The parameter values are $h=0.26 \mu \mathrm{m}, w_{i}=0.15 \mu \mathrm{m}, a=0.35 \mu \mathrm{m}, d^{\prime}=0.15 \mu \mathrm{m}, d=0.20 \mu \mathrm{m}$, and $w=1.0224 \mu \mathrm{m}$.

Figure 2 (c) plots the dispersion relation for the lowest odd-symmetry band in our sample device in the region near the band edge. Frequency is plotted in units of $2 \pi c / a$ and wave vector is plotted in units of $2 \pi / a$. For $n_{\mathrm{Si}}=3.4845$ and $n_{\mathrm{SiO}_{2}}=1.45$, the first band gap was $23.5 \%$ of the mid-gap frequency for odd modes with respect to the mirror plane of the structure ( $\vec{E}$ mostly parallel to the substrate). For $n_{\mathrm{Si}}=3.4845$, the band edge frequency was calculated to be $\omega_{\mathrm{be}}=0.227182 \pi c / a$ for the lowest band, or $\omega_{\mathrm{be}} / 2 \pi=190 \mathrm{THz}(\lambda=1.54 \mu \mathrm{m})$. For $n_{\mathrm{Si}}^{\prime}=3.45(\Delta n / n=-0.01)$, the band edge shifted to $\omega_{\mathrm{be}}=0.228802 \pi c / a$, or $\omega_{\mathrm{be}} / 2 \pi=200 \mathrm{THz}(\lambda=1.53 \mu \mathrm{m})$.

In previous work, we have calculated the relevant figures of merit to characterize the performance of the device. ${ }^{16}$ In particular, we have shown that the so-called sensitivity of the device, defined as the ratio of the change in fractional time delay to the fractional change in index, increases as the operating frequency approaches the band edge. Moreover, using analytical arguments, we have shown that this increase is a general feature of periodically-grated devices operated near the photonic band edge.

\section{MODAL SYMMETRIES}

The dispersion relation shown above in Figure 2 (c) focuses on one particular guided mode of the grating in the region near the band edge. To clarify the properties of the guided modes, we discuss the modal symmetries in the device and our related nomenclature in further detail. Much confusion can arise from the differing nomenclatures used in the guided-wave and photonic-crystal literature. (Of particular issue is the use of the terms "TE" and "TM" to describe mode polarizations, which can vary between different works.) To avoid ambiguity, we classify different guided modes by their symmetry with respect to any mirror-plane symmetries of the structure. ${ }^{19}$ The structure of Figure 2 (a) and (b) is only symmetric with respect to a single mirror plane, indicated by the dashed line in Figures 3(a) and 3(b), which show a end- and top-view of our structure. Due to the presence of the substrate, the structure is not symmetric with respect to the $y=0$ plane. This is in contrast to the well-studied case of suspended, photonic-crystal slab structures. ${ }^{20}$

Guided modes can be either even or odd with respect to the mirror plane of the structure. Modal symmetry relates to the way in which the electric-field vector transforms under mirror operations. ${ }^{19}$ In brief, a mode that is even with respect to the $z=0$ plane has individual field components with the following symmetries. $E_{x}$ and 


\section{(a) end view}

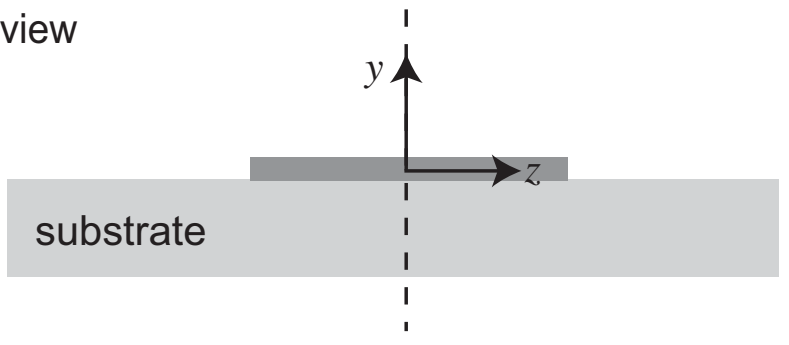

(b) top view

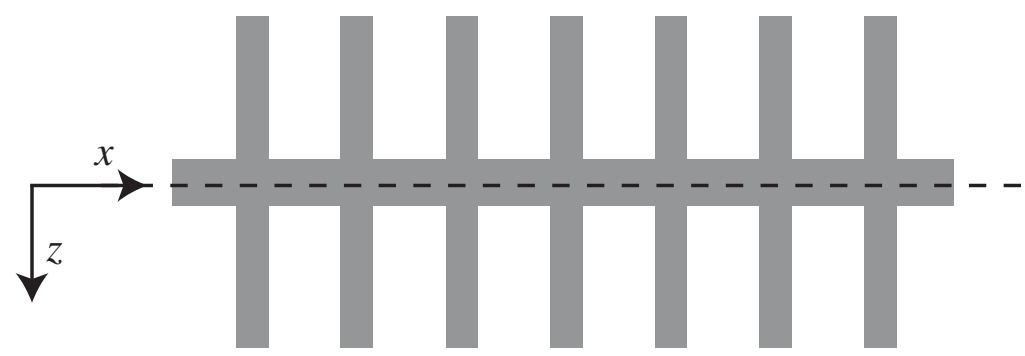

Figure 3. Mirror symmetry of the grating structure. The dashed line indicates the mirror plane. (a) End view. (b) Top view.

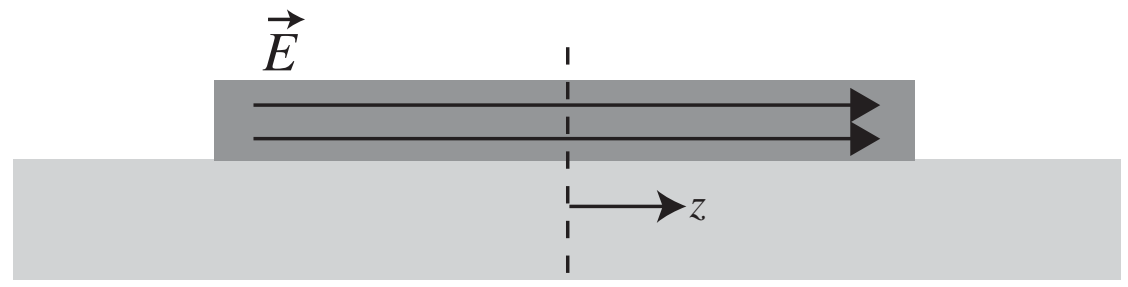

Figure 4. An electric field plot consistent with odd vector symmetry with respect to the mirror plane indicated by the dashed line.

$E_{y}$ are both even with respect to the mirror plane, while $E_{z}$ is odd. A mode that is odd with respect to the $z=0$ mirror plane has $E_{x}$ and $E_{y}$ odd, while $E_{z}$ is even. For completeness, we add that the individual magnetic field components transform differently than the electric field. Even modes have $H_{x}$ and $H_{y}$ odd, while $H_{z}$ is even; odd modes have $H_{x}$ and $H_{y}$ even, while $H_{z}$ is odd. ${ }^{19}$

The consequence of this discussion is that a mode that has an $\vec{E}$-field predominantly parallel to the substrate and no nodes in the center, as illustrated in (Figure 4) has an even $E_{z}$ component, but has odd vector symmetry, corresponding to the mode symmetry for the dispersion diagram in Figure 2 (c) above.

\section{COMPLETE BAND STRUCTURE}

Figure 5 shows the complete dispersion relation of the grating structure. Bands were computed for $n_{\mathrm{Si}}=3.4845$. Odd-symmetry bands are shown by solid, black lines. The lowest odd band, or fundamental mode, is the same as shown in Figure 2 (c), and is the only odd guided mode over its entire frequency range. Even bands are shown as dashed lines.

Figure 6 shows the $E_{z}$ component of the electric field for the odd modes at $k=\pi / a$. The left and right set of panels show the end- and top-views, respectively. Grey represents a value of zero, while white and black correspond to negative and positive values. The lowest (fundamental) mode, labeled o1, has no vertical nodes 


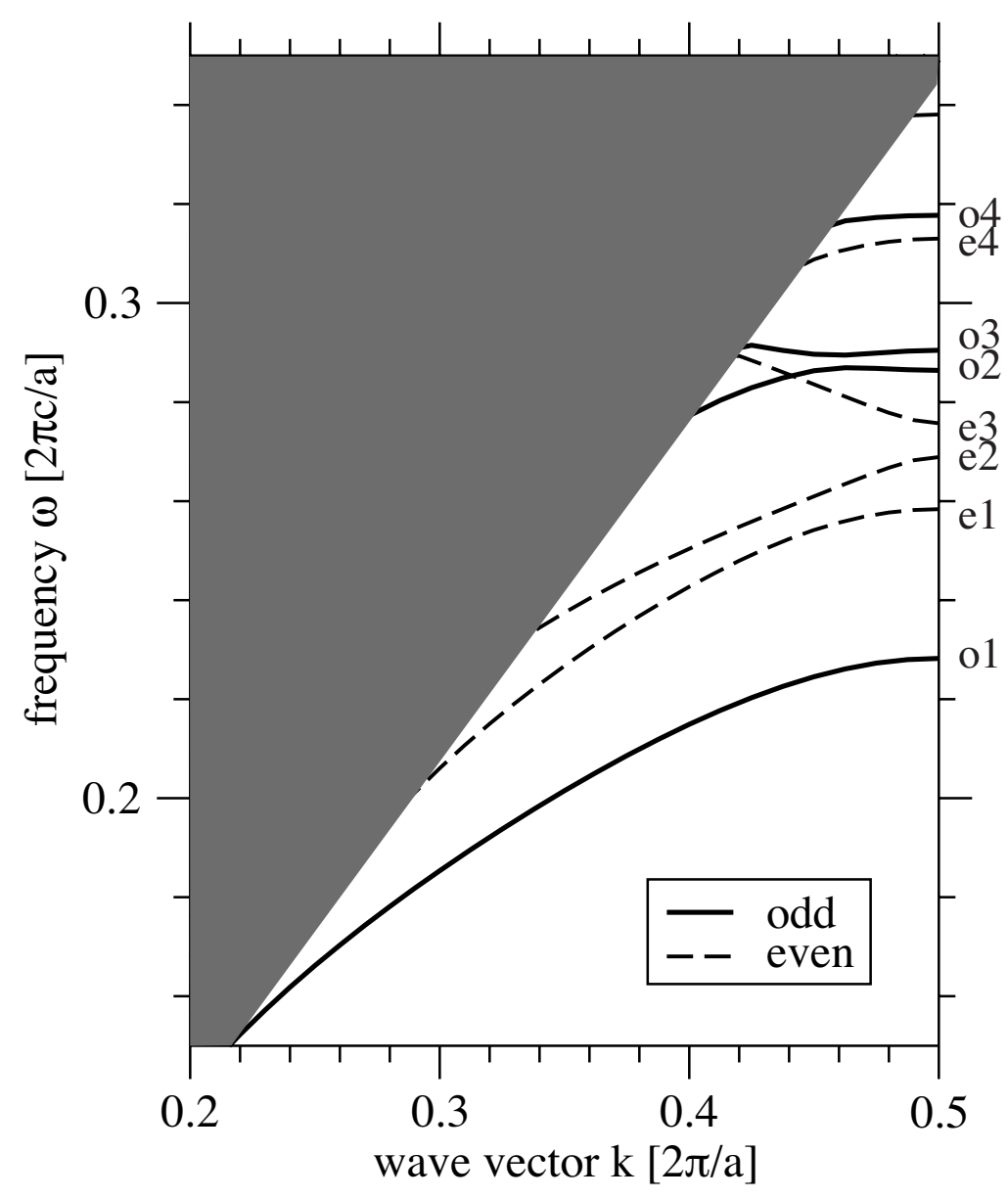

Figure 5. Complete band structure of the grating structure, showing multiple bands with both even and odd symmetry.

in $E_{z}$ (see end view) and is concentrated in the thicker regions of the grating (see top view). The o2 mode also has no nodes, and is concentrated in the thinner regions of the grating and may be interpreted as the folded fundamental mode. The o3 band has one vertical node in $E_{z}$, and $E_{z}$ is mostly concentrated in the thicker regions of the grating. The 04 band has both a vertical node and a horizontal node. The shape of the second and third odd bands in Figure 5 results from an avoided crossing between the o2 and o3 modes. Due to the presence of the substrate, there is no horizontal symmetry plane through the grating. As a result, o2 and o3 can weakly couple despite their nearly-orthogonal vertical profiles (compare end views), and a weak splitting results.

In our previous work, we have described how two gratings with oppositely-curved bands can be cascaded to achieve dispersion compensation. ${ }^{16}$ Note that for this purpose, a second grating structure must be designed in which the folded, fundamental mode is separated in frequency from other bands.

Figure 7 shows the $E_{z}$ component of the even modes at $k=\pi / a$. The lowest even mode, labeled e1, has no vertical nodes and a single horizontal node. $E_{z}$ is largely concentrated in the thicker regions of the grating. The e2 mode has one horizontal and one vertical node, and is also concentrated in the thicker regions of the grating. The e 3 mode is formed from the folding of the e 2 mode, as is apparent from the band structure in Figure 5 and is concentrated in the thinner regions of the grating. The e4 mode has one vertical and three horizontal nodes and is concentrated in the thicker regions of the grating. 

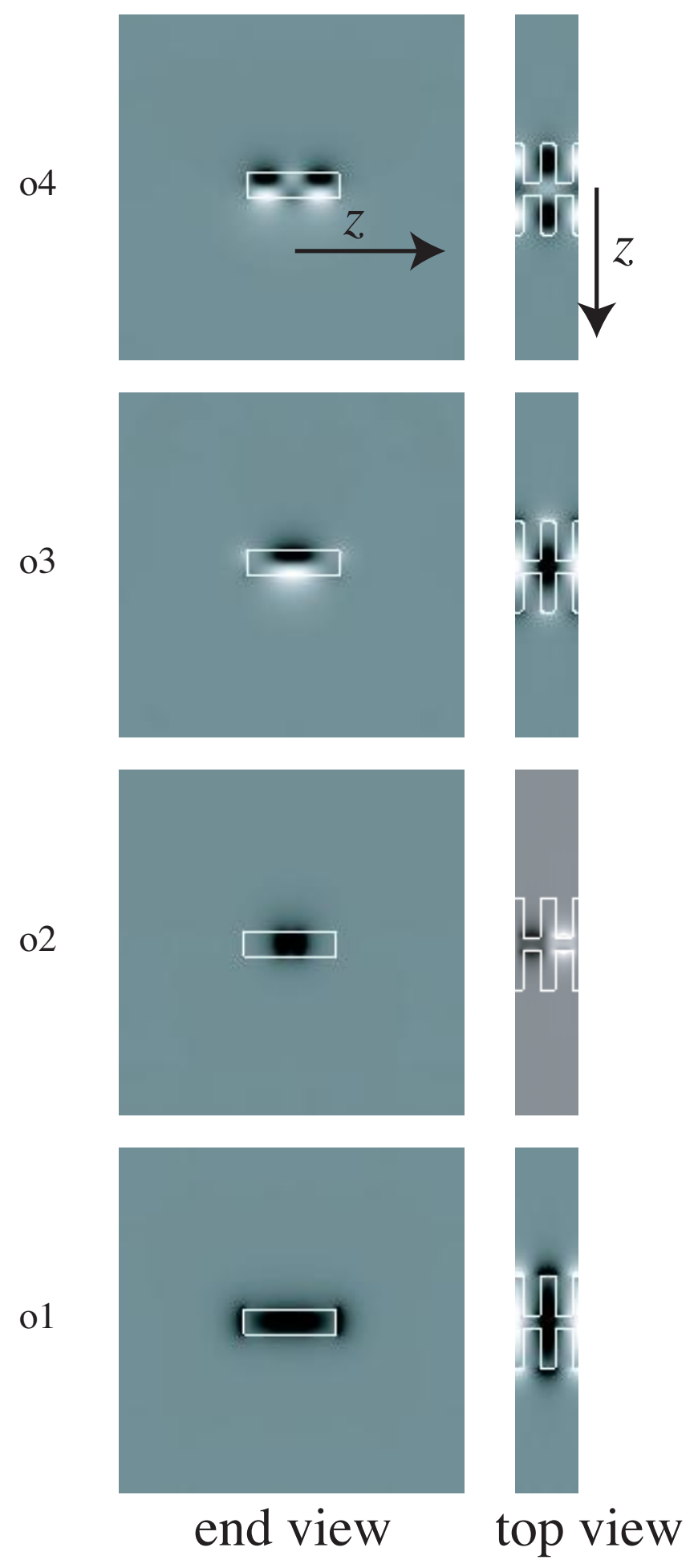

Figure 6. Odd guided modes. Plotted is the $E_{z}$ component of each mode, with white, grey, and black corresponding to negative, zero, and positive values. 

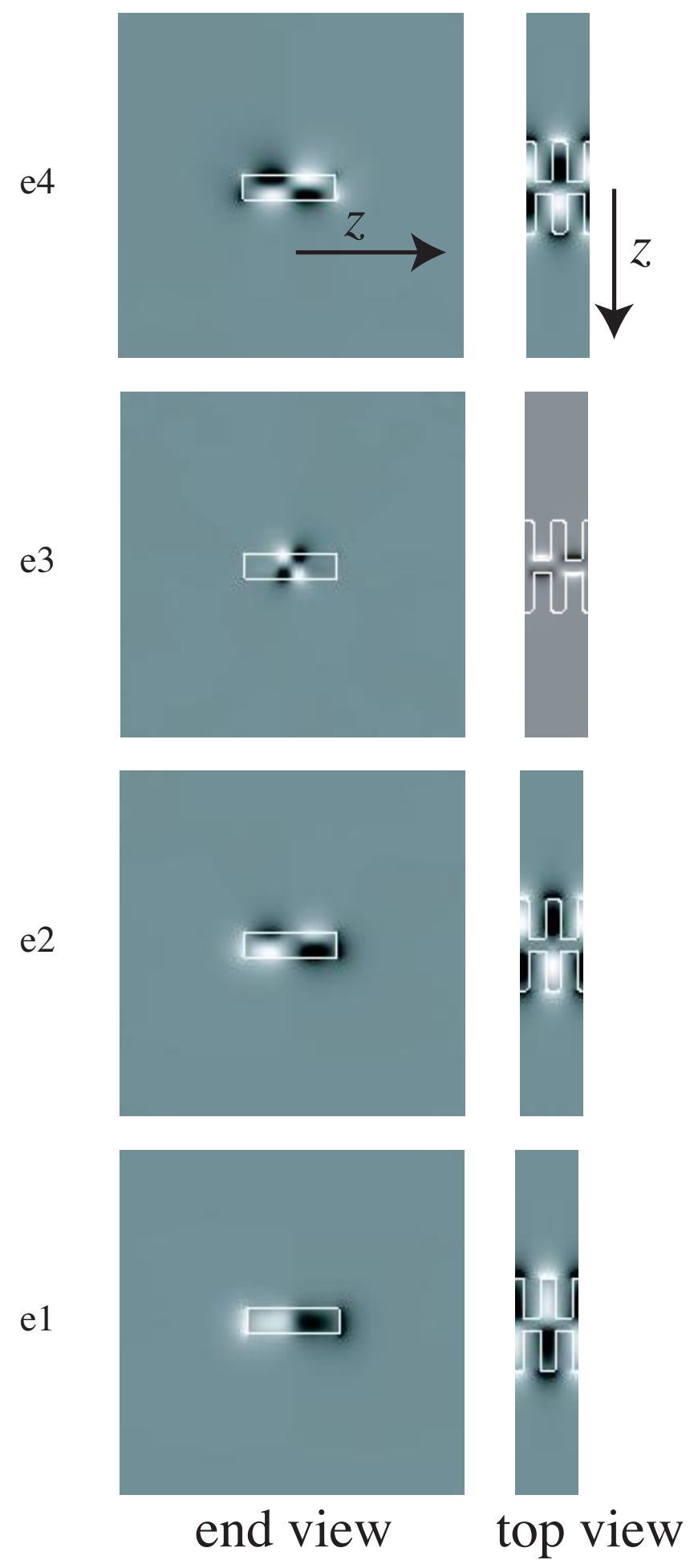

Figure 7. Even guided modes. Plotted is the $E_{z}$ component of each mode, with white, grey, and black corresponding to negative, zero, and positive values. 


\section{CONCLUSION}

In this manuscript, we have elaborated on the mode symmetry and band structure of silicon-on-insulator gratings proposed for tunable time delay applications. The descriptions contained herein should aid in the understanding of our previous publication and aid the future design of a class of integrated, tunable time-delay devices.

\section{REFERENCES}

1. G. Lenz, B. J. Eggleton, C. K. Madsen, and R. E. Slusher, "Optical delay lines based on optical filters," IEEE J. Quantum Electron. 37, pp. 525-532, Apr. 2001.

2. R. Ramaswami and K. N. Sivarajan, Optical Networks: A Practical Perspective, Academic Press, London, 1998.

3. M. S. Rasras, C. K. Madsen, M. A. Cappuzzo, E. Chen, L. T. Gomez, E. J. Laskowski, A. Griffin, A. WongFoy, A. Gasparyan, A. Kasper, J. LeGrange, and S. S. Patel, "Integrated resonance-enhanced variable delay lines," IEEE Photonics Tech. Lett. 17, pp. 834-6, Apr. 2005.

4. Z. Wang and S. Fan, "Compact all-pass filters in photonic crystals as the building block for high-capacity optical delay lines," Phys. Rev. E 68, p. 066616, December 2003.

5. A. Yariv, Y. Xu, R. K. Lee, and A. Scherer, "Coupled-resonator optical waveguide: a proposal and analysis," Opt. Lett. 24, pp. 711-713, 1999.

6. S. Mookherjea and A. Yariv, "Coupled resonator optical waveguides," IEEE Journal of Selected Topics in Quantum Electronics 8(3), pp. 448-456, 2002.

7. J. Liu, B. Shi, D. Zhao, and X. Wang, "Optical delay in defective photonic bandgap structures," J. Opt. A.: Pure Appl. Opt. 4, pp. 636-639, Oct. 2002.

8. S. Nishikawa, S. Lan, N. Ikeda, Y. Sugimoto, H. Ishikawa, and K. Asakawa, "Optical characterization of photonic crystal delay lines based on one-dimensional coupled defects," Opt. Lett. 27, pp. 2079-2081, Dec. 2004.

9. H. Altug and J. Vučković, "Two-dimensional coupled photonic crystal resonator arrays," Appl. Phys. Lett. 84, pp. 161-163, Jan. 2004.

10. H. Altug and J. Vučković, "Experimental demonstration of the slow group velocity of light in two-dimensional coupled photonic crystal microcavity arrays," Appl. Phys. Lett. 86, p. 111102, 2005.

11. M. F. Yanik, W. Suh, Z. Wang, and S. Fan, "Stopping light in a waveguide with an all-optical analog of electromagnetically induced transparency," Physical Review Letters 93, p. 233903, December 2004.

12. M. F. Yanik and S. Fan, "Stopping light all optically," Physical Review Letters 92, p. 083901, February 2004.

13. M. Scalora, R. J. Flynn, S. B. Reinhardt, R. L. Fork, M. J. Bloemer, M. D. Tocci, C. M. Bowden, H. S. Ledbetter, J. M. Bendickson, and R. P. Leavitt, "Ultrashort pulse propagation at the photonic band edge: Large tunable group delay with minimal distortion and loss," Phys. Rev. E 54, pp. R1078-1081, Aug. 1996.

14. D. Mori and T. Baba, "Dispersion-contolled optical group delay device by chirped photonic crystal waveguides," Appl. Phys. Lett. 85, pp. 1101-1103, Aug. 2004.

15. D. Mori and T. Baba, "Wideband and low dispersion slow light by chirped photonic crystal coupled waveguide," Opt. Express 13, pp. 9398-9408, Nov. 2005.

16. M. L. Povinelli, S. G. Johnson, and J. D. Joannopoulos, "Slow-light, band-edge waveguides for tunable time delays," Opt. Express 13, pp. 7145-7159, 2005.

17. J. T. Hastings, M. H. Lim, J. G. Goodberlet, and H. I. Smith, "Optical waveguides with apodized sidewall gratings via spatial-phase locked electron-beam lithography," J. Vac. Sci. Technol. B 20, pp. 2753-2757, Nov/Dec 2002.

18. Y. A. Vlasov, M. O'Boyle, H. F. Hamann, and S. J. McNab, "Active control of slow light on a chip with photonic crystal waveguides," Nature 438, pp. 65-69, November 2005.

19. J. D. Joannopoulos, R. D. Meade, and J. N. Winn, Photonic Crystals: Molding the Flow of Light, Princeton Univ. Press, 1995.

20. S. G. Johnson, S. Fan, P. R. Villeneuve, J. D. Joannopoulos, and L. A. Kolodziejski, "Guided modes in photonic crystal slabs," Phys. Rev. B 60, pp. 5751-5758, Aug. 1999. 